Research Paper

\title{
Radiotherapy and Sorafenib in the Management of Patients with Hepatocellular Carcinoma Have Led to Improved Survival: A Single Center Experience
}

\author{
Assaf Moore1, Michal Cohen-Naftaly3,2, Ofer Benjaminov2,4, Marius Braun²,3, Assaf Issachar2,3, Eitan Mor 2,5, \\ Anna Tovar ${ }^{2,6}$, Michal Sarfaty,1,2, Noa Gordon ${ }^{1}$, and Salomon M. Stemmer ${ }^{1,2}$ \\ 1. Institute of Oncology, Davidoff Center, Rabin Medical Center, Petah Tikva, Israel; \\ 2. Sackler Faculty of Medicine, Tel Aviv University, Tel Aviv, Israel; \\ 3. Liver Institute; Rabin Medical Center, Petah Tikva, Israel; \\ 4. Department of Imaging; Rabin Medical Center, Petah Tikva, Israel; \\ 5. Department of Organ Transplantation; Rabin Medical Center, Petah Tikva, Israel; \\ 6. Department of Pathology, Rabin Medical Center, Petah Tikva, Israel. \\ $\triangle$ Corresponding author: Stemmer SM, M.D., Institute of Oncology, Davidoff Center, Rabin Medical Center, Petah-Tiqva, Israel. Telephone: 972-3-9378101; Fax: \\ 972-3-9478114; e-mail: stemmer@post.tau.ac.il.
}

( ) Ivyspring International Publisher. Reproduction is permitted for personal, noncommercial use, provided that the article is in whole, unmodified, and properly cited. See http://ivyspring.com/terms for terms and conditions.

Received: 2015.12.16; Accepted: 2016.03.22; Published: 2016.04.29

\begin{abstract}
Background \& Aims: Hepatocellular Carcinoma (HCC) is the sixth most common malignancy and the third most common cause of cancer mortality worldwide. We aimed to assess the effect of novel treatment options on the survival of HCC patients.

Methods: This retrospective study included all HCC patients diagnosed between 2000 and 2013 referred to the Davidoff center and treated by a multidisciplinary team.

Results: The analysis included 321 patients (median age, 64 years; 74.8\% males; $74.1 \%$ viral carriers; $76.0 \%$ cirrhosis; $56.7 \%$ diagnosis at an early stage). The estimated hazard ratio by multivariate analysis for the effect of the period of diagnosis (2007-2013 vs. $2000-2006)$ on survival was $0.72(95 \% \mathrm{Cl}$ : $0.54-0.96 ; p=0.027)$. There was no difference in the distribution by $C P$ score, by $B C L C$ stage at diagnosis or in the proportion of patients undergoing surgical procedures (liver transplantation or resection). In the later time frame, there was a significant decrease in the proportion of patients undergoing percutaneous treatments ( $14.6 \%$ vs. $4.2 \%, p=0.004)$ and embolization $(46.9 \%$ vs. $24.6 \%, p=0.001)$, and a significant increase in radiotherapy $(1.5 \%$ vs. $8.4 \%, p=0.009)$ and treatment with sorafenib $(6 \%$ vs. $18.3 \%$, $\mathrm{p}=0.002$ ).

Conclusion: Technological/pharmaceutical innovations have led to advancement in HCC treatment. Since there was no significant difference in the proportion of patients undergoing surgical procedures during the evaluated timeframe, the improved survival may stem from better management of advanced stage patients by a multidisciplinary team.
\end{abstract}

Key words: hepatocellular carcinoma, liver transplantation, sorafenib, retrospective study.

\section{Introduction}

Hepatocellular Carcinoma (HCC) is the sixth most common malignancy, and the third most common cause of cancer mortality worldwide [1]. HCC has well-defined risk factors with the most significant risk factor being cirrhosis [2,3]. Surveillance is indicated for early detection of HCC in high-risk patients [4].
Unique staging methods have been developed, such as the Barcelona-Clinic Liver Cancer staging classification (BCLC), which relates to the Child Pugh (CP) Score of liver function, as well as to the patient's performance status (PS) as evaluated by the Eastern Cooperative Oncology Group score [5,6].

Therapeutic options recommended based on the 
BCLC staging system reflect prognosis and survival. BCLC stage A patients are candidates for surgical procedures, such as resection or transplantation $[2,6]$. For unresectable lesions or in patients with poorer PS with sufficient hepatic reserve, percutaneous methods or imaging-guided tumor ablation are the treatment of choice [2,7,8]. Arterial chemoembolization is first-line palliative treatment for unresectable or multi-focal HCC in the absence of vascular invasion or metastases, and is recommended for BCLC stage B patients $[2,6,8]$. Radiotherapy was limited in the past by the risk of radiation-induced liver disease [2]; however, due to advances in radiation technology, at present, doses of up to 80-90 Gy can be safely delivered to the tumor, with minimal exposure of uninvolved liver tissue [9].

Progress in the field of advanced HCC seems to be slow and modest, and clinicians are faced with growing numbers of patients presenting with advanced or recurrent incurable HCC. BCLC stage C patients are candidates for systemic treatment or participating in clinical trials; whereas BCLC stage D patients require best supportive care [6]. The role of systemic chemotherapy is limited due to its lack of efficacy and toxicity [2,10]. Sorafenib, a tyrosine kinase inhibitor, was shown to improve OS in HCC patients with $\mathrm{CP}$ score A (10.7 vs. 7.9 months) and is the only approved agent in this setting $[2,11]$. Due to the lack of effective treatments in patients with advanced HCC, new treatments and technologies are being evaluated.

In this study we examined the effects of novel technologies and the trends in treatment selection, as seen in a tertiary center on patients suffering from HCC over time.

\section{Patients and methods}

\section{Patients and data collection}

The study included all HCC patients who were referred for treatment and follow-up to the Institute of Oncology, Davidoff Center, Rabin Medical Center between 2000 and 2013.

Data were collected from medical records and included demographics, social history, habits, comorbidities, viral status, history of cirrhosis and underlying liver disease, tumor parameters at diagnosis, PS, detailed treatment information and response, survival, and cause of death.

HCC diagnosis was established according to international guidelines, biopsy, or radiology. Stratification was performed according to the BCLC classification.

Treatment outcomes were assessed by imaging studies and tumor markers. Disease-free survival
(DFS) was defined as the period of time between a potentially curative treatment such as transplant or resection and the first imaging study indicating recurrence. Progression-free survival (PFS) was defined as the period of time between a non-curative treatment such as TACE or sorafenib and the first imaging study recorded indicating disease progression. Treatment-related death was defined as death within 30 days of treatment. Cause of death was determined using medical records.

\section{Statistical analysis}

Data were analyzed using the Statistical Package for the Social Sciences 22.0 (SPSS) at a significance level of $0.05 . \chi^{2}$ or Fisher's exact tests were used for categorical data, and the Mann-Whitney- $U$ test or student's T test were used for continuous data.

Survival was estimated using the Kaplan-Meier method and Cox's regression analysis.

\section{Results}

\section{Patients' characteristics}

Charts of all 373 HCC patients evaluated at the Institute of Oncology, Davidoff Center between 2000 and 2013 were reviewed; 52 patients, who were treated elsewhere and received a single consultation were excluded. Thus, the study included 321 HCC patients: $74.8 \%$ male, median age at diagnosis 64.2 (29.7-89.5) years with male patients being diagnosed at a younger age than female patients (63.2 vs. 67.7 years, $p=0.002) ; 75.7 \%$ had viral hepatitis and the majority (76.0)\% had underlying cirrhosis (specifically, $2.0 \%$ of the cohort had a history of primary biliary cirrhosis, $1.2 \%$ had primary sclerosing cholangitis, and $0.3 \%$ had autoimmune hepatitis). 209 (65.1\%) had CP score A, and 182 (56.7\%) were BCLC stage A. $57.9 \%$ of patients had tissue diagnosis (Table 1).

\section{Survival analysis}

Median OS for all patients (Fig. 1) was 22.7 (17.4-28.1) months. The difference in OS between all stages was statistically significant $(p<0.0001)$. Patients diagnosed at an early stage (BCLC stage A) had a median OS of 43.3 months; those diagnosed at an intermediate (BCLC stage $B, n=41$ ), advanced (BCLC stage $C, n=59$ ), and end-stage (BCLC stage $D$, $\mathrm{n}=29$ ), had a median OS of 13.9, 9.9, and 3.4 months, respectively. In patients for whom stage at diagnosis was unknown $(n=10)$, median OS was 36.5 months.

No statistical difference was observed in OS based on viral hepatitis status (14.6, 26.3. 28.1, and 25.4 months for HBV carriers, HCV carriers, carriers of both, and non-carriers, respectively $(\mathrm{p}=0.14)$. 
Table 1. Demographics and baseline characteristics for 321 patients with HCC.

\begin{tabular}{|c|c|}
\hline Characteristics & All Patients $(\mathrm{n}=321)$ \\
\hline Median age at diagnosis, yr (range) & $64.2(29.7-89.5)$ \\
\hline Male, $\mathrm{n}(\%)$ & $240(74.8)$ \\
\hline \multicolumn{2}{|l|}{ Habits, n (\%) } \\
\hline Smoking & $133(41.4)$ \\
\hline Alcohol consumption & $37(11.5)$ \\
\hline IV drug use & $6(1.9)$ \\
\hline \multicolumn{2}{|l|}{ Comorbidities, n (\%) } \\
\hline Hypertension & $150(46.7 \%)$ \\
\hline COPD & $24(7.5 \%)$ \\
\hline Diabetes Mellitus & $112(34.9 \%)$ \\
\hline Ischemic Heart Disease & $47(14.6 \%)$ \\
\hline \multicolumn{2}{|l|}{ Liver disease, $\mathrm{n}(\%)$} \\
\hline Cirrhosis & $244(76.0)$ \\
\hline Unknown & $7(2.2)$ \\
\hline \multicolumn{2}{|l|}{ Viral hepatitis, $\mathrm{n}(\%)$} \\
\hline Carriers of any viral hepatitis & $243(75.7)$ \\
\hline $\mathrm{HCV}$ only & $147(45.8)$ \\
\hline HBV only & $85(26.5)$ \\
\hline $\mathrm{HCV}$ and $\mathrm{HBV}$ & $11(3.4)$ \\
\hline \multicolumn{2}{|c|}{ Tumor Markers, $\mathrm{n}$ elevated/n available (\%) ,median (range) } \\
\hline AFP & $\begin{array}{l}209 / 281(74.4 \%), 49.0 \\
(0.8-100 \mathrm{~K}) \mathrm{ng} / \mathrm{ml}\end{array}$ \\
\hline CEA & $\begin{array}{l}37 / 120(37.4 \%), 2.75(0.4-33.0) \\
\mathrm{U} / \mathrm{ml}\end{array}$ \\
\hline CA19-9 & $\begin{array}{l}64 / 123(52.0 \%), 36.1 \\
(0.1-9231.0) \mathrm{U} / \mathrm{ml}\end{array}$ \\
\hline \multicolumn{2}{|l|}{ Child-Pugh, n (\%) } \\
\hline A & $209(65.1)$ \\
\hline B & $72(22.4)$ \\
\hline $\mathrm{C}$ & $28(8.7)$ \\
\hline Unknown & $12(3.7)$ \\
\hline \multicolumn{2}{|l|}{ Histology, n (\%) } \\
\hline Tissue Sample acquired & $186(57.9)$ \\
\hline HCC NOS & $42(22.6)$ \\
\hline Well-differentiated HCC & $40(21.5)$ \\
\hline Well-to-moderately differentiated HCC & $19(10.2)$ \\
\hline Moderately-differentiated HCC & $57(30.6)$ \\
\hline Moderately-to-poorly differentiated HCC & $10(5.4)$ \\
\hline Poorly-differentiated HCC & $6(3.2)$ \\
\hline Clear Cell HCC & $2(1.1)$ \\
\hline Fibrolamellar HCC & $1(0.5)$ \\
\hline \multicolumn{2}{|l|}{ Stage at diagnosis, $\mathrm{n}(\%)$} \\
\hline BCLC A & $182(56.7)$ \\
\hline BCLC B & $41(12.8)$ \\
\hline BCLC C & $59(18.4)$ \\
\hline BCLC D & $29(9.0)$ \\
\hline Unknown & $10(3.1)$ \\
\hline \multicolumn{2}{|l|}{ Extrahepatic Metastases, $\mathrm{n}(\%)$} \\
\hline At Diagnosis & $18(5.6)$ \\
\hline Metastases in disease progression & $59(18.4)$ \\
\hline Unknown & $6(1.9)$ \\
\hline
\end{tabular}

\section{Survival benefit by period of diagnosis}

Of the 321 patients in our cohort, 130 were diagnosed between 2000 and 2006, and 191 between 2007 and 2013. Median (range) OS was 18.9 (12.3-25.5) months for the former, and 24.3 (16.1-32.5) months for the latter (Fig. 2). In a multivariate analysis (adjusted for gender, age at diagnosis, stage at diagnosis, and hepatitis status), the estimated hazard ratio (HR) for the effect of the period of diagnosis (2007-2013 vs. 2000-2006) on OS was 0.72 (95\% CI, 0.54-0.96, p = 0.027 , with no significant differences in the distribution of BCLC stage or CP score at diagnosis by period of diagnosis.

\section{Treatment outcomes}

Liver transplantation was performed in 62 patients; 33 patients had bridging therapies prior to liver transplantation. Transplant related mortality was $8 \%$. Ten patients $(16.1 \%)$ recurred; including 2 of the 7 transplanted patients who did not meet the Milan Criteria (28.6\%) and 3 of 4 patients $(75 \%)$ in whom stage at diagnosis was unknown. 47/51 (92\%) of transplanted patients who met the Milan Criteria remained disease free. Kaplan-Meier estimate for median DFS (Fig. 3) and OS was not reached (>100 months) with no difference related to transplant year.

In total, 65 tumor resections were performed, including 56 primary resections and 9 resections of a recurrent mass (either after liver transplantation or after a former resection). Nine patients had bridging treatments prior to resection. Surgery-related mortality was $6.2 \%$ and 37 patients (56.9\%) recurred. Median DFS was 22.5 months (Fig. 3) and estimated median survival time from resection was 91.6 months.

Twenty-eight patients underwent percutaneous treatments. Patients who were treated or were eligible for transplantation or resection were not included in this group; 27 patients had RFA, 1 patient had percutaneous ethanol injection. Twenty-one patients (75\%) recurred. Treatment-related mortality was $7.1 \%$, median PFS was 19.7 months (Fig. 3), and estimated median survival time following the procedure was 53.5 months.

Overall, 133 arterial embolization treatments (doxorubicin 52\%, cisplatin $16.5 \%$ ) were performed. Eighteen patients received serial treatments without disease progression in the intervals. In these cases, PFS was calculated as the time between the first treatment and the time of disease progression. Treatment-related mortality was $1.5 \%$. Median PFS was 6.8 months (Fig. 3), and estimated median survival time following the procedure was 18.0 months.

Twenty-four patients received radiotherapy to liver masses. Radiotherapy methods included intensity-modulated radiation therapy (IMRT) and stereotactic body radiation therapy (SBRT). These patients were incompatible with other methods due to liver function, tumor size, and location. In 6 patients, radiotherapy was followed by liver transplantation, without disease progression in the interval. The remaining 18 patients had a median PFS of 11.9 
months (Fig. 3), and median survival time following radiotherapy of 10.5 months. Treatment- related mortality was $8.3 \%$. Two patients died of sepsis during radiotherapy; one case was unrelated to the tumor or diseased liver.

Forty-three patients received sorafenib. No treatment-related mortality was recorded. Median PFS was 3.8 months (Fig. 3), and estimated median survival was 13.9 months (Fig. 4).
A total of 41 patients enrolled in clinical trials. For 9 patients, the clinical trial treatment was their

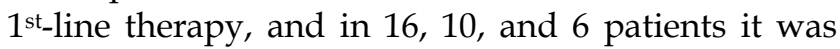
their $2^{\text {nd }}$, $3^{\text {rd }}$ and $4^{\text {th }}-$ line respectively. Some patients participated in more than one trial. In these cases, PFS was calculated for each trial separately. No treatment-related mortality was recorded. Median PFS was 5.5 months (Fig. 3), and estimated median survival following clinical trials was 9.0 months.

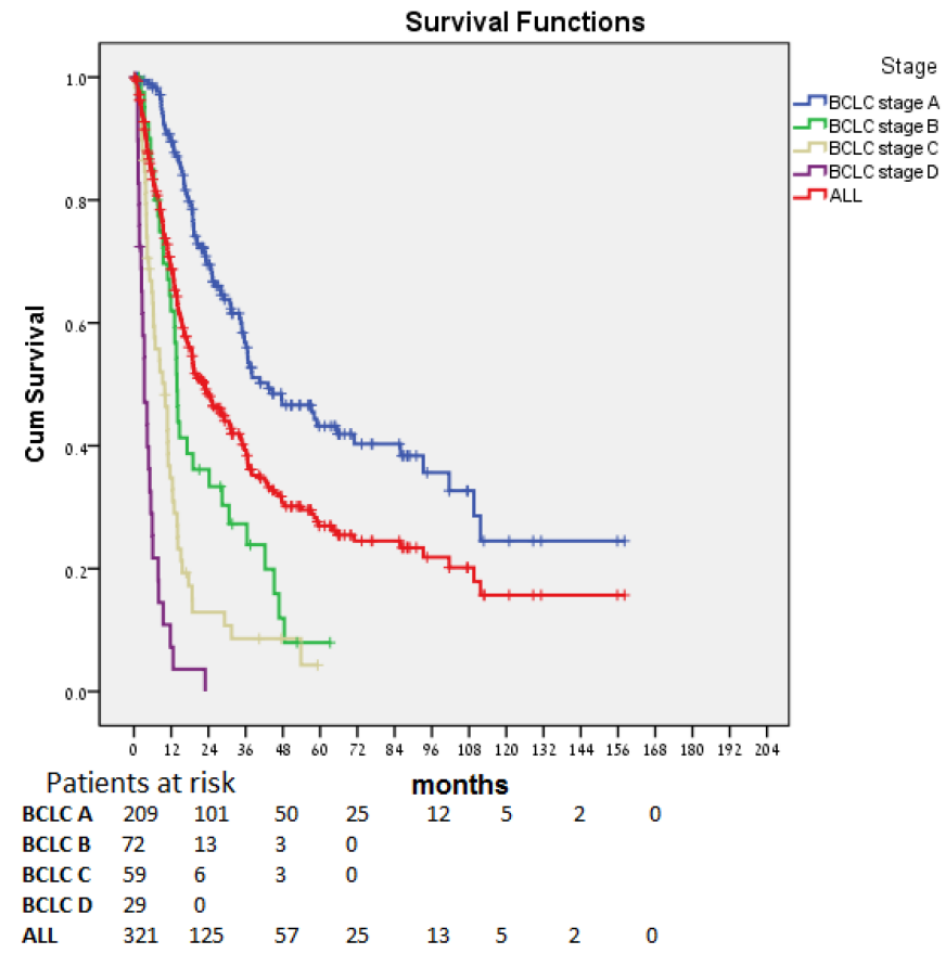

Figure 1. Overall survival over time for the entire cohort and by stage at diagnosis.

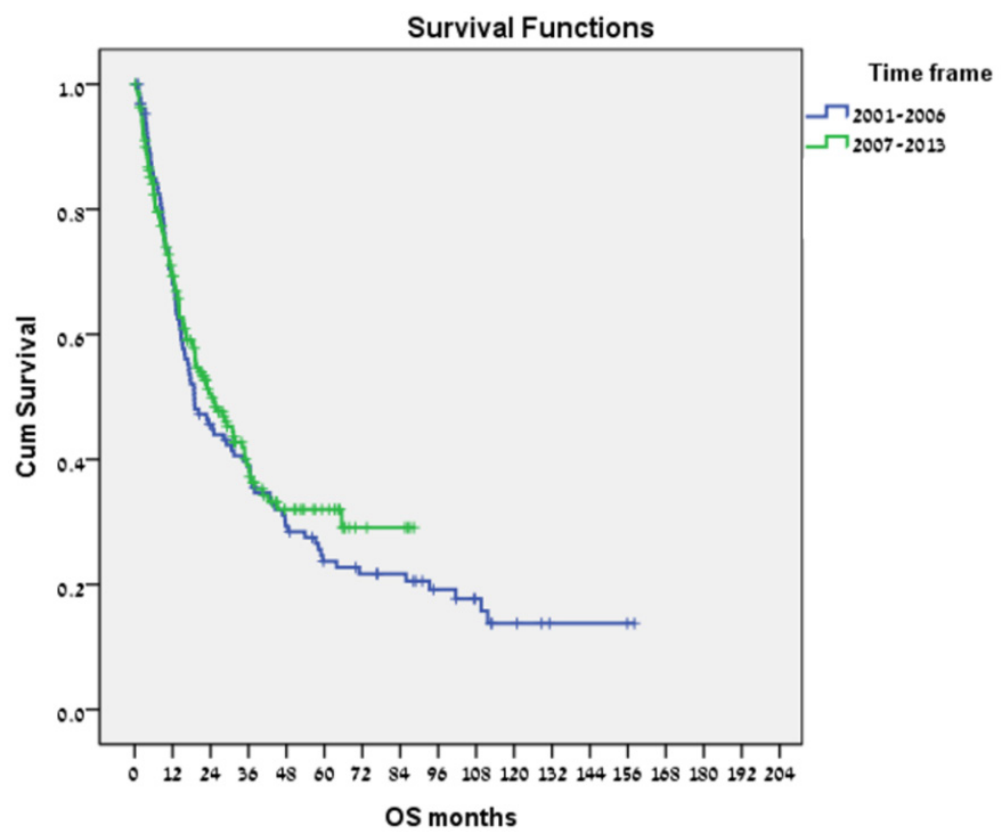

Figure 2. Overall survival by period of diagnosis (2000-2006 vs 2007-2013). 


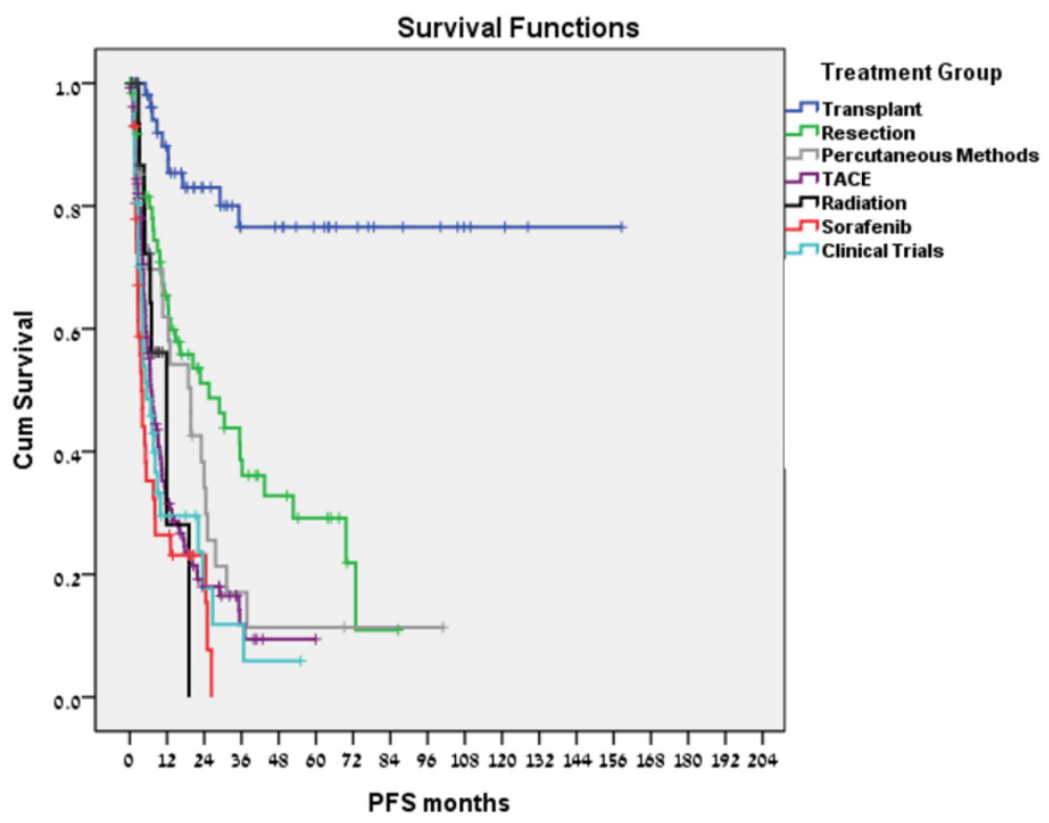

Figure 3. PFS for various treatment modalities.

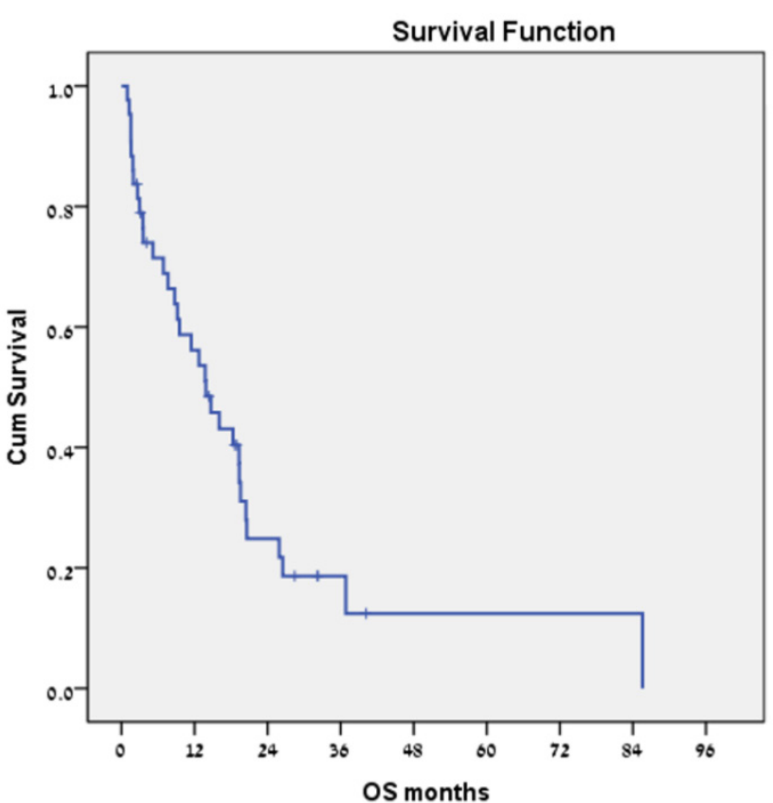

Figure 4. Overall survival for the subgroup of patients with hepatocellular carcinoma who were treated with sorafenib $(n=43)$.

\section{Discussion}

We found that the period of diagnosis influenced OS, with patients diagnosed in the last 7 years (2007-2013) experiencing significantly better OS than patients treated in the 7 preceding years (2000-2006) (HR of $0.72, p=0.027$ ) with no difference in the distribution by $\mathrm{CP}$ score or by BCLC stage at diagnosis. These differences were not the result of early detection as there was no difference in the proportion of patients undergoing surgical procedures (liver transplantation or resection). In the later time frame, there was a significant decrease in the proportion of patients undergoing percutaneous treatments $(14.6 \%$ vs. $4.2 \%, p=0.004)$ and embolization $(46.9 \%$ vs. $24.6 \%, p=0.001)$, and a significant increase in radiotherapy $(1.5 \%$ vs. $8.4 \%, \mathrm{p}=$ 0.009 ) and treatment with sorafenib ( $6 \%$ vs. $18.3 \%, \mathrm{p}=$ 0.002 ). Therefore, the longer survival probably stems from improved management of advanced-stage patients.

Moreover, we observed shorter median OS for early stage of disease (BCLC stages A and B) and longer for advanced disease (BCLC stage $C$ ) in comparison to Cillo et al group [12]. The shorter median OS of BCLC stage A patients (43.3 vs. 53.0 months) and B (13.9 vs 16 months) may be explained by a lower availability of transplantable organs in Israel, as well as borderline BCLC B patients in terms of PS, being eventually analyzed as BCLC C. The longer median OS observed for patients with advanced disease may be related to an abundance of clinical trials, palliative treatments, and multidisciplinary supportive care.

The recurrence rate of $16.1 \%$ in transplanted patients is consistent with the finding by D'amico et al who reported $18 \%$ recurrence rate in their series of 479 transplanted patients [13] and we found a similar 5 year survival rate $(>70 \%)$ as reported by others when the Milan Criteria are met $[13,14,15]$.

As expected, we observed a higher recurrence rate $(56.9 \%)$ in patients undergoing liver resection and an estimated median survival time from resection of 91.6 months consistent with studies demonstrating 
5-year survival for resected CP A patients who have a localized tumor of $>70 \%$, and $50-60 \%$ in patients with portal hypertension or multifocal tumors $[15,16,17]$. There is increasing evidence for survival advantage exciding the BCLC stage [17]. In our study the results are probably affected by the advanced BCLC stage of some of our resected patients, the large size of some of the resected tumors (up to $10 \mathrm{~cm}$ ), and selection bias of patients referred to and treated at the Institute of Oncology.

As for the locoregional therapy, the recurrence rate was $75 \%$ for RFA-treated patients and the median DFS was 19.7 months, different from the outcomes reported by Lee et al [18] in a series of 162 patients

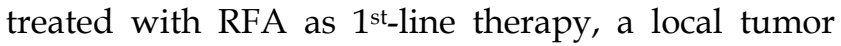
progression rate of $14.5 \%$ at 5 years, overall 5-year survival and recurrence-free survival of $67.9 \%$ and $25.9 \%$ respectively.

Arterial embolization had a median PFS of 6.8 months. As this modality is indicated for patients with multifocal disease, progression of the untreated masses is likely to decrease the PFS. Median survival time following the procedure was 18.0 months, consistent with known data for BCLC stage B patients $[7,8]$.

The group of patients treated with radiotherapy to the liver mass showed an estimated median PFS of 11.9 months. Bujold et al [19], in their study involving102 patients unsuitable for standard locoregional therapies and treated with SBRT (doses: 24-54 Gy), showed a local control at 1 year of $87 \%$ and median OS of 17 months. Thus, our study, albeit having a small group of radiotherapy-treated patients, suggests that radiotherapy may be a reasonable alternative for patients who are not candidates for invasive treatments.

Interestingly, we found that sorafenib-treated patients had a median survival of 13.8 months, longer than the median OS (8.5-9.5 months) in a recent publication, and in despite of a median PFS of 3.8 months, similar to a recent phase III study comparing clinical outcomes in patients with advanced HCC treated with sorafenib plus either erlotinib or placebo [20]. This reflects better patient selection, as well as better treatment options after progression on sorafenib including participation in clinical trials and palliative treatments. Patients enrolled in clinical trials had a median PFS of 5.5 months, and an estimated median survival following clinical trials of 9.0 months. The majority of patients had been previously treated with other modalities. As an example, in February 2009, an 89-years old patient was diagnosed with BCLC stage B disease. This patient enrolled in a clinical trial and remained progression free for almost 5 years. This further demonstrates the importance of clinical trials for advanced disease when alternatives are limited.

This study is limited by its retrospective design. Also, this study was not population-based, but focused on HCC patients who were referred to, treated and followed at the Institute of Oncology. Thus, some patients who had surgery/ transplantation at Rabin Medical Center may have been treated/followed up elsewhere, thereby influencing our survival data. In addition, the quality of the data within the medical records may vary, as these data were documented by multiple treating physicians.

In conclusion, our data showed that technological and pharmaceutical innovations have led to improved OS in HCC patients during the last 7 years, as a decline in locoregional treatments and increase in radiotherapy and treatment with sorafenib may be the explanation for the improvement in survival. Examining the effects of these innovations should be elucidated in prospective trials, and may resolve some of the important clinical questions in HCC management.

\section{Abbreviations}

AFP, Alpha-fetoprotein; BCLC, Barcelona-Clinic Liver Cancer Staging Classification; CA19-9, cancer antigen 19-9; CEA, carcinoembryonic antigen; $C P$, Child Pugh; DFS, disease-free survival; HBV, hepatitis $B$ virus; HCC, hepatocellular carcinoma; $\mathrm{HCV}$, hepatitis $\mathrm{C}$ virus; $\mathrm{HR}$, hazard ratio; OS, overall survival; PFS, progression-free survival; PS, performance status; RFA, radiofrequency ablation; TACE, trans arterial chemo-embolization

\section{Acknowledgements}

We gratefully acknowledge our colleagues for their contribution to the formation of this study, as well as for their dedicated care of the patients. We also thank the staff of the Institute of Oncology at the Davidoff Center, the Liver Institute, the Department of Organ Transplantation, the Department of Imaging, and the Department of Pathology at Rabin Medical Center.

\section{Conflict of interest}

The authors declare no conflict of interest.

\section{References}

[1] Jemal A, Bray F, Center MM, Ferlay J, Ward E, Forman D, et al. Global cancer statistics. CA Cancer J Clin. 2011;61(2):69-90.

[2] Forner A, Llovet JM, Bruix J, et al. Hepatocellular carcinoma. Lancet. 2012 Mar 31;379(9822):1245-55

[3] El-Serag HB, Rudolph KL. Hepatocellular carcinoma: Epidemiology and molecular carcinogenesis. Gastroenterology. 2007 Jun;132(7):2557-76.

[4] Bruix J, Shreman M. Management of hepatocellular carcinoma: an update. Hepatology. 2011 Mar; 53(3): 1020-1022. 
[5] Okuda K, Ohtsuki T, Obata H, et al. Natural history of hepatocellular carcinoma and prognosis in relation to treatment: study of 850 patients. Cancer. 1985 Aug 15;56(4):918-28.

[6] Llovet JM, Brú C, Bruix J, et al. Prognosis of hepatocellular carcinoma: the BCLC staging classification. Semin Liver Dis. 1999;19(3):329-38.

[7] Vogl TJ, Naguib NN, Zangos S, et al. Review on transarterial chemoembolization in hepatocellular carcinoma: Palliative, combined, neoadjuvant, bridging, and symptomatic indications. Eur J Radiol. 2009 Dec;72(3):505-16.

[8] Takayasu K, Arii S, Ichida T, et al. Prospective cohort study of transarterial chemoembolization for unresectable hepatocellular carcinoma in 8,510 patients. Gastroenterology. 2006 Aug;131(2):461-9.

[9] Tse RV, Guha C, Dawson LA, et al. Conformal radiotherapy for hepatocellular carcinoma. Crit Rev Oncol Hematol. 2008 Aug:67(2):113-23.

[10] Thomas MB, O'Beirne JP, Johnson P, et al. Systemic therapy for hepatocellular carcinoma: cytotoxic chemotherapy, targeted therapy and immunotherapy. Ann Surg Oncol. 2008 Apr;15(4):1008-14.

[11] De Lope CR, Tremosini S, Bruix J, et al. Management of HCC. J Hepatol. 2012;56 Suppl 1:S75-87

[12] Cillo U, Vitale A, Zanus G, et al. Prospective validation of the Barcelona Clinic Liver Cancer staging system. J Hepatol. 2006 Apr; 44(4): 723-31.

[13] D'Amico F, Schwartz M, Thung S, et al. Predicting recurrence after liver transplantation in patients with hepatocellular carcinoma exceeding the up-to-seven criteria. Liver Transpl. 2009 Oct;15(10):1278-87.

[14] Mazzaferro V, Llovet JM, Mariani L, et al. Predicting survival after liver transplantation in patients with hepatocellular carcinoma beyond the Milan criteria: a retrospective, exploratory analysis. Lancet Oncol. 2009 Jan;10(1):35-43.

[15] Llovet JM, Schwartz M, Mazzaferro V, et al. Resection and liver transplantation for hepatocellular carcinoma. Semin Liver Dis. 2005;25(2):181-200.

[16] Poon RT, Fan ST, Wong J et al. Difference in tumor invasiveness in cirrhotic patients with hepatocellular carcinoma fulfilling the Milan criteria treated by resection and transplantation: impact on long-term survival. Ann Surg. 2007 Jan;245(1):51-8.

[17] Vitale A, Burra P, Spolverato G, et al. Survival benefit of liver resection for patients with hepatocellular carcinoma across different Barcelona Clinic Liver Cancer stages: a multicentre study. J Hepatol. 2015 Mar;62(3):617-24.

[18] Lee DH, Lee JM, Kim YJ, et al. Radiofrequency ablation of hepatocellular carcinoma as first-line treatment: long-term results and prognostic factors in 162 patients with cirrhosis. Radiology. 2014 Mar 30;270(3):900-9.

[19] Bujold A, Massey CA, Wong RK, et al. Sequential phase I and II trials of stereotactic body radiotherapy for locally advanced hepatocellular carcinoma. J Clin Oncol. 2013 May 1;31(13):1631-9.

[20] Zhu AX, Rosmorduc O, Carrilho FJ, et al. SEARCH: a phase III, randomized, double-blind, placebo-controlled trial of sorafenib plus erlotinib in patients with advanced hepatocellular carcinoma. J Clin Oncol. 2015 Feb 20;33(6):559-66. 